\title{
An analytical MHD wind model with latitudinal dependences obtained using separation of the variables
}

\author{
J. J. G. Lima ${ }^{1,2}$, E. R. Priest ${ }^{3}$, and K. Tsinganos ${ }^{4}$ \\ 1 Centro de Astrofísica, Universidade do Porto, Rua das Estrelas, 4150-762 Porto, Portugal \\ 2 Departamento de Matemática Aplicada, Faculdade de Ciências, Universidade do Porto, Portugal \\ 3 School of Mathematical and Computational Sciences, University of St. Andrews, St. Andrews KY16 9SS, \\ Scotland, UK \\ e-mail: eric@mcs.st-and.ac.uk \\ 4 Department of Physics, University of Crete and FORTH, PO Box 2208, 71003 Heraklion, Crete, Greece \\ e-mail: tsingan@physics.uoc.gr
}

Received 12 May 2000 / Accepted 28 February 2001

\begin{abstract}
A new class of analytical 2-D solutions of the full set of the steady magnetohydrodynamic (MHD) equations, describing an axisymmetric helicoidal magnetized outflow originating from a rotating central object, is presented. The solutions are systematically obtained via a nonlinear separation of the variables in the momentum equation. The analysis yields three parameters which measure the anisotropy in the latitudinal distribution of various flow quantities. Topologically, the wind speed is controlled by an X-type critical point that acts to filter out a single wind-type branch and the Alfvén singularity. The solutions can be regarded as an extension outside the equatorial plane of the Weber \& Davis (1967) model of magnetized winds but with a variable polytropic index.
\end{abstract}

Key words. magnetohydrodynamics (MHD) - plasmas - Sun: magnetic fields - solar wind - stars: winds, outflows - ISM: jets and outflows

\section{Introduction}

Recent detailed observations of the solar wind (e.g., via Ulysses and SoHO, Feldman et al. 1996) and other astrophysical outflows (e.g., via the Hubble Space Telescope, Biretta 1996) have highlighted the need to provide a satisfactory description of cosmic MHD outflows which deals adequately and simultaneously with their: (i) spatial 2-D (or 3-D) character, (ii) nonlinear dynamics, (iii) detailed energy balance and (iv) time-dependent nature. At the moment this task is far from being completed and still remains a challenge for the future. The present treatment is not going to change this fact that we do not have available to this day a satisfactory MHD solar/stellar wind description. Hence, by necessity, in the present paper we shall constrain our efforts only to the above items (i)-(ii).

The first models of astrophysical outflows were polytropic and 1-D (Parker 1958, 1963), or, all forces perpendicular to the equatorial plane were ignored (Weber \& Davis 1967). The discovery of flows through coronal holes called for the investigation of quasi-two dimensional polytropic models at first (Kopp \& Holzer 1976; Habbal \& Tsinganos 1983) and fully 2-D numerical models later

Send offprint requests to: J. J. G. Lima, e-mail: jlima@astro.up.pt
(Pneuman \& Kopp 1971; Steinolfson et al. 1982; Nerney \& Suess 1975; Sakurai 1985). Recently, time-dependent studies of thermally and/or magneto-centrifugally-driven polytropic wind-type outflows provided new and promising results in relation to the question of the degree of collimation of the outflow (Ouyed \& Pudritz 1997; Keppens \& Goedbloed 1999; Ustyugova et al. 1999; Tsinganos \& Bogovalov 2000; Krasnopolsky et al. 1999). Non-polytropic wind modelling on the other hand, with energy and momentum addition and finite thermal conductivity has been increasingly used because observations have highlighted the fact that the acceleration of the solar wind in highspeed streams (Feldman et al. 1996; Giordano et al. 2000) does imply energy and momentum addition in the solar corona (Leer \& Holzer 1980; Steinolfson 1988; Suess et al. 1996; Hansteen et al. 1997; Wang et al. 1998).

A fully 2-D MHD modelling of steady hydromagnetic wind-type outflows in open magnetic fields has been introduced (Low \& Tsinganos 1986; Tsinganos \& Low 1989) with a variable polytropic index $\gamma$ wherein it was shown that the density needs to decrease with latitude for an accelerated wind. This is because a dipolar magnetic field needs to be kept open by a pressure that must decrease towards the pole. If the density does not vary with latitude, there is a smaller pressure gradient to drive the flow near the pole, exactly where the magnetic field is open to 
allow the wind to escape. The resulting acceleration is too low, since gravity dominates, and the flow does not reach a high enough terminal speed. The only way out is to allow the density to increase with latitude, faster than the pressure does (Hu \& Low 1989). Subsequently, solutions of the MHD equations with a latitudinally-dependent density and a helicoidal geometry of the streamlines were analysed (Tsinganos \& Trussoni 1991, hereafter referred to as TT91), but the latitudinal dependence of the different quantities was assumed a priori. Under the same assumptions, this work was later continued (Trussoni \& Tsinganos 1993; Trussoni et al. 1997). Other analytical solutions which have used similar assumptions have concentrated on the asymptotic analysis and its links to the intitial boundary conditions and were able to derive a criterion for the collimation of winds into jets (Sauty \& Tsinganos 1994; Sauty et al. 1999).

In this paper we shall deduce from the governing MHD equations via a nonlinear separation of the variables the latitudinal variation of the density and other relevant physical quantities of the wind, instead of adopting a priori for them a specific form, as it was done in TT91 and in subsequent papers of that series. This shall also further extend previous work (Lima \& Priest 1993, hereafter referred as Paper I), which deduced a general class of solutions of the hydrodynamic equations relevant to stellar winds with a helicoidal geometry. Section 2 is devoted to the method of solution, Sect. 3 deals with its parametric study and finally Sect. 4 ends with a discussion of the results.

\section{Method of solution}

\subsection{Basic equations and assumptions}

We shall seek 2-D solutions of the set of steady MHD equations describing the dynamical interaction of an inviscid, compressible and highly conducting plasma with an axially symmetric magnetic field created by a central rotating object. These include Maxwell's equation for the divergence of the magnetic field, the induction equation in the limit of large conductivity and the equations for conservation of mass and momentum (Priest 1982). The pressure is related to the density and temperature through the classical ideal gas law $p=\left(2 k_{\mathrm{B}} / m\right) \rho T$ while the temperature is obtained from an energy conservation equation such as the first law of thermodynamics, including a heating/cooling term.

The system of MHD equations can be closed if the heating function is functionally related to the thermodynamic variables and the flow field. One such simple example which has been widely used in the literature is when we have a polytropic law between pressure and density. The alternative approach that we follow here is to find some other functional relationship between the heating rate, pressure, density and flow speed such that the variables are separable.
The obvious choice of coordinate system appropriate to this type of problem is that of spherical polar coordinates $(r, \theta, \phi)$, with $\theta$ as the co-latitude. With axisymmetry, in order to make the above equations more tractable from an analytical point of view, the simplifying assumption will be made that the meridional components of both the velocity and magnetic field can be neglected (i.e. $V_{\theta}=$ $\left.B_{\theta}=0\right)$. Under this assumption the projection of the field lines on the poloidal plane corresponds to radial lines. Note that it has been recently shown that this assumption of radiality is a good one, at least in the case of the solar wind (Wang et al. 1998; Tsinganos \& Bogovalov 2000).

Then, the system of MHD equations can be re-written explicitly in the following way:

$$
\begin{aligned}
& \frac{\partial}{\partial r}\left(B_{\mathrm{r}} r^{2}\right)=0 \\
& \frac{\partial}{\partial r}\left(r V_{\phi} B_{\mathrm{r}}-r V_{\mathrm{r}} B_{\phi}\right)=0 \\
& \frac{\partial}{\partial r}\left(\rho r^{2} V_{\mathrm{r}}\right)=0 \\
& \rho V_{\mathrm{r}} \frac{\partial V_{\mathrm{r}}}{\partial r}-\rho \frac{V_{\phi}^{2}}{r}=-\frac{\partial p}{\partial r}-\frac{B_{\phi}^{2}}{4 \pi r}-\frac{B_{\phi}}{4 \pi} \frac{\partial B_{\phi}}{\partial r}-\frac{\rho G M}{r^{2}} \\
& \rho V_{\phi}^{2} \frac{\cos \theta}{\sin \theta}=\frac{\partial p}{\partial \theta}+\frac{B_{\mathrm{r}}}{4 \pi} \frac{\partial B_{\mathrm{r}}}{\partial \theta}+\frac{B_{\phi}^{2}}{4 \pi} \frac{\cos \theta}{\sin \theta}+\frac{B_{\phi}}{4 \pi} \frac{\partial B_{\phi}}{\partial \theta} \\
& \rho V_{\mathrm{r}} \frac{\partial V_{\phi}}{\partial r}+\rho \frac{V_{\mathrm{r}} V_{\phi}}{r}=\frac{B_{\mathrm{r}} B_{\phi}}{4 \pi r}+\frac{B_{\mathrm{r}}}{4 \pi} \frac{\partial B_{\phi}}{\partial r}
\end{aligned}
$$

\subsection{A technique based on a separation of the variables}

At this stage, we introduce a second simplifying assumption, namely that the variables are separable in $r$ and $\theta$. This will transform the above system of partial differential equations into a system of ordinary differential equations which are analytically more tractable.

Denoting by $r_{0}$ the radius at the base of the atmosphere, we can non-dimensionalise all quantities with respect to their values at this reference level. In particular we set $R=r / r_{0}$. Using the assumption of separation of variables, we can write the radial velocity, the azimuthal velocity and the azimuthal magnetic field as, respectively,

$V_{\mathrm{r}}(R, \theta)=V_{0} Y(R) v_{\mathrm{r}}(\theta)$

$V_{\phi}(R, \theta)=V_{1} A(R) v_{\phi}(\theta)$,

$B_{\phi}(R, \theta)=B_{1} M(R) b_{\phi}(\theta)$,

where $V_{0}, V_{1}$ and $B_{1}$ correspond to their reference values. Note that, at this stage, the functions $v_{\mathrm{r}}(\theta), v_{\phi}(\theta)$ and $b_{\phi}(\theta)$ are completely arbitrary. From Eqs. (1), (3) we must also have

$B_{\mathrm{r}}(R, \theta)=B_{0} \frac{b_{\mathrm{r}}(\theta)}{R^{2}}, \quad \rho(R, \theta)=\rho_{0} \frac{g(\theta)}{Y R^{2}}$,

with $B_{0}$ and $\rho_{0}$ as the values of the radial magnetic field and density at the reference level, while $b_{\mathrm{r}}(\theta)$ and $g(\theta)$ are 
as yet to be determined. Note that the radial magnetic field has a monopole geometry modified by the presence of $b_{\mathrm{r}}(\theta)$.

Using the same technique described in Paper I, let us eliminate the pressure term between the $r$ - and $\theta$-components of the momentum equation, Eqs. (4), (5), by differentiating the first one with respect to $\theta$ and the second one with respect to $r$, and then adding. The resulting expression is

$$
\begin{gathered}
\frac{\partial}{\partial \theta}\left(\rho \frac{V_{\phi}^{2}}{r}\right)-\frac{\partial}{\partial \theta}\left(\rho V_{\mathrm{r}} \frac{\partial V_{\mathrm{r}}}{\partial r}\right)-\frac{1}{4 \pi} \frac{\partial}{\partial \theta}\left(\frac{B_{\phi}^{2}}{r}\right) \\
-\frac{1}{4 \pi} \frac{\partial}{\partial \theta}\left(B_{\phi} \frac{\partial B_{\phi}}{\partial r}\right)-\frac{\partial}{\partial \theta}\left(\frac{\rho G M}{r^{2}}\right) \\
-\frac{\partial}{\partial r}\left(\rho V_{\phi}^{2} \frac{\cos \theta}{\sin \theta}\right)+\frac{1}{4 \pi} \frac{\partial}{\partial r}\left(B_{\mathrm{r}} \frac{\partial B_{\mathrm{r}}}{\partial \theta}\right) \\
+\frac{1}{4 \pi} \frac{\partial}{\partial r}\left(B_{\phi}^{2} \frac{\cos \theta}{\sin \theta}\right)+\frac{1}{4 \pi} \frac{\partial}{\partial r}\left(B_{\phi} \frac{\partial B_{\phi}}{\partial \theta}\right)=0
\end{gathered}
$$

Under the assumption of separation of variables, the above equation will be transformed into an ordinary differential equation involving functions of $R$ alone. For that purpose, the functions of $\theta$ in each term will be set proportional to each other. We should keep in mind, however, that this last procedure may not give us the most general separable solution in the sense that some terms could be also set proportional to the sum of the others. Thus, from the last two terms, we may write

$b_{\phi} \frac{\mathrm{d} b_{\phi}}{\mathrm{d} \theta}=\epsilon b_{\phi}^{2} \frac{\cos \theta}{\sin \theta}$

which implies that

$$
\frac{\mathrm{d}}{\mathrm{d} \theta}\left(\frac{b_{\phi}^{2}}{\sin ^{2 \epsilon} \theta}\right)=0,
$$

and so

$$
b_{\phi}(\theta)=\sin ^{\epsilon} \theta
$$

where $\epsilon$ is an arbitrary constant. Note that the constant of integration has been set to unity, without loss of generality. Any other value for this constant can be incorporated into the radial dependence of $B_{\phi}(R, \theta)$. In what follows, the choice of the constants of proportionality between different terms as well as the constants of integration will be made so as to obtain the simplest possible solutions, without loss of generality. Comparing the 7th and 8th terms, we can put

$b_{\mathrm{r}} \frac{\mathrm{d} b_{\mathrm{r}}}{\mathrm{d} \theta}=\mu \epsilon b_{\phi}^{2} \frac{\cos \theta}{\sin \theta}$,

which implies

$b_{\mathrm{r}}(\theta)=\sqrt{1+\mu \sin ^{2 \epsilon} \theta}$,

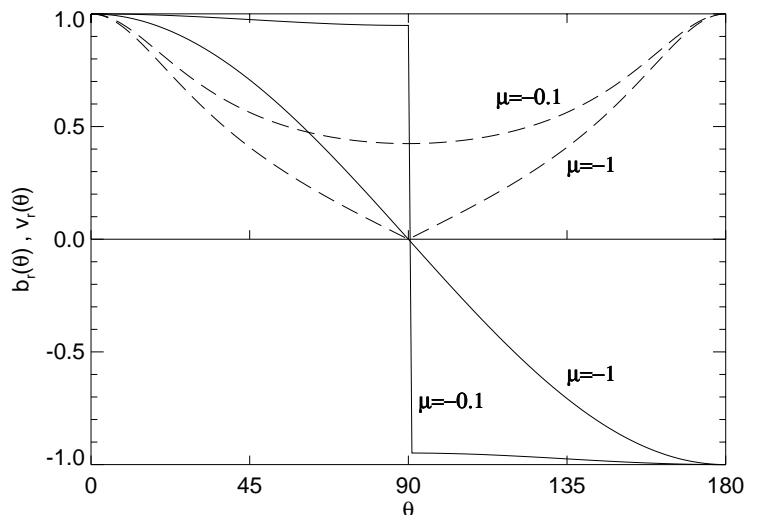

Fig. 1. Co-latitudinal dependence of the radial magnetic field $b_{\mathrm{r}}(\theta)$ (solid line) and of the radial velocity (dashed line) for $\epsilon=1$ and $\delta=4$

where $\mu$ is a second arbitrary constant. From the 5 th and 8th terms in Eq. (11) we may also obtain

$\frac{\mathrm{d} g}{\mathrm{~d} \theta}=2 \delta \epsilon b_{\phi}^{2} \frac{\cos \theta}{\sin \theta}$,

thus resulting in

$g(\theta)=1+\delta \sin ^{2 \epsilon} \theta$,

in which $\delta$ is the third arbitrary constant. As for the 6 th and 8 th terms we can write

$g v_{\phi}^{2}=b_{\phi}^{2}$

or, equivalently,

$v_{\phi}(\theta)=\frac{\sin ^{\epsilon} \theta}{\sqrt{1+\delta \sin ^{2 \epsilon} \theta}}$.

Finally, from the $\phi$-component of the momentum equation, Eq. (6), for the separation in $R$ and $\theta$ to work we must have

$g v_{\mathrm{r}} v_{\phi}=b_{\mathrm{r}} b_{\phi}$,

giving

$v_{\mathrm{r}}(\theta)=\sqrt{\frac{1+\mu \sin ^{2 \epsilon} \theta}{1+\delta \sin ^{2 \epsilon} \theta}}$.

Note that from the four remaining terms in Eq. (11) 1st, 2nd 3rd and 4th - we obtain identities, provided the proportionality constants are chosen accordingly.

We have thus deduced general $\theta$-dependences for the density and hydromagnetic field, under the assumption of separation of variables, Eqs. (14), (16), (18), (20) and (22), in terms of three arbitrary constants: $(\delta, \epsilon, \mu)$. These parameters effectively control the anisotropy in the outflow, in the magnetic field and in the density distribution.

First, the parameter $\delta$ is related to the ratio of the density at the equator $(\theta=\pi / 2)$ to that at the pole $(\theta=$ $0)$ and so the higher it is, the more the density distribution deviates from the spherically symmetric case $(\delta=0)$. 
Second, the parameter $\mu$ is related to the ratio of the radial kinetic energy density at the equator to that at the pole. Finally, the parameter $\epsilon$ controls the width of the profile of the speed and density for some fixed variation between pole and equator.

Note that in the TT91 model, the simplest possible forms of $b_{\mathrm{r}}(\theta), g(\theta)$ and $v_{\mathrm{r}}(\theta)$ were chosen a priori such that they were able to simulate existing observations of the solar wind. Their expressions constitute a special case of the forms deduced in this work, corresponding to $\epsilon=1$ and $\mu=-1$.

\subsection{Angular momentum and angular velocity}

Returning to the $\phi$-component of the momentum equation, Eq. (6), it can be manipulated to give

$\frac{\partial}{\partial r}\left(r \sin \theta V_{\phi}-r \sin \theta \frac{B_{\mathrm{r}} B_{\phi}}{4 \pi \rho V_{\mathrm{r}}}\right)=0$.

The solution of this equation introduces a function of $\theta$ of the form

$L(\theta)=r \sin \theta V_{\phi}-r \sin \theta \frac{B_{\mathrm{r}} B_{\phi}}{4 \pi \rho V_{\mathrm{r}}}$,

which is the total angular momentum per unit mass loss carried away by the wind, along each flow line $\theta=$ const (see Weber \& Davis 1967). The first term on the right-hand side of Eq. (24) is the angular momentum carried by the advection of the flow while the second term represents the torque associated with the magnetic stresses. Analogously, the solution to the induction equation, Eq. (2), introduces another function of $\theta$ of the form

$\Omega(\theta)=\frac{1}{r \sin \theta}\left(V_{\phi}-B_{\phi} \frac{V_{\mathrm{r}}}{B_{\mathrm{r}}}\right)$,

which corresponds to the angular velocity of the roots of the field lines on the surface of the central object.

The azimuthal components of the velocity and magnetic fields can now be obtained via these two functions $L(\theta)$ and $\Omega(\theta)$. After some straightforward manipulation we arrive at:

$V_{\phi}=\frac{r_{0}}{Y_{*}}\left(\frac{Y_{*}-Y}{1-M_{\mathrm{A}}^{2}}\right) R \sin \theta \Omega(\theta)$,

$B_{\phi}=\frac{B_{0} r_{0}}{V_{0} Y_{*}}\left(\frac{R^{2} / R_{*}^{2}-1}{1-M_{\mathrm{A}}^{2}}\right) \frac{\Omega(\theta) \sin \theta \sqrt{1+\delta \sin ^{2 \epsilon} \theta}}{R}$,

where $M_{\mathrm{A}}^{2}=\left(V_{\mathrm{r}} / V_{\mathrm{A}}\right)^{2}$ is the radial Alfvén Mach number which corresponds to the ratio of the radial velocity, $V_{\mathrm{r}}$, to the radial Alfvénic velocity, $V_{\mathrm{A}}=B_{\mathrm{r}} / \sqrt{4 \pi \rho}$, and $\left(R_{*}, Y_{*}\right)$ is the point for which $M_{\mathrm{A}}=1$. At this point, $Y_{*} R_{*}^{2}=$ $\left(M_{\mathrm{A}}^{0}\right)^{-2}$, where

$M_{\mathrm{A}}^{0}=\frac{V_{0}}{V_{0}^{\mathrm{A}}}$

is the ratio of the radial velocity to the Alfvénic radial velocity at the base of the wind. A physical interpretation of the radial Alfvén Mach number $M_{\mathrm{A}}$ can be (a)

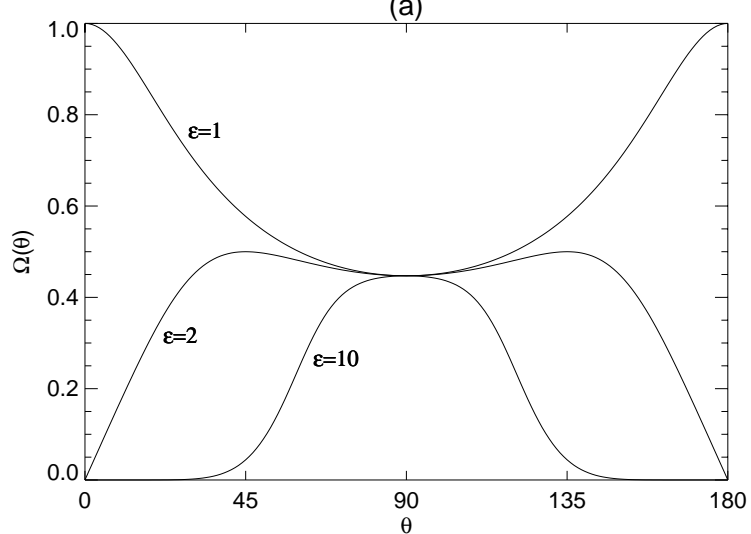

(b)

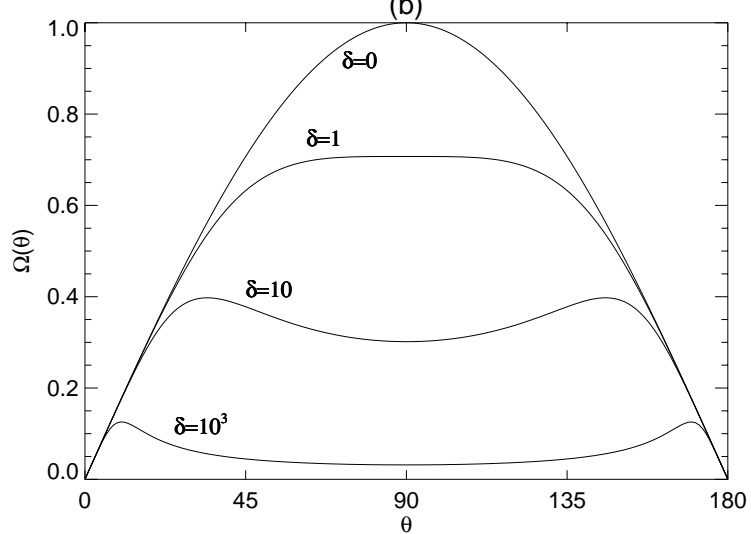

(c)

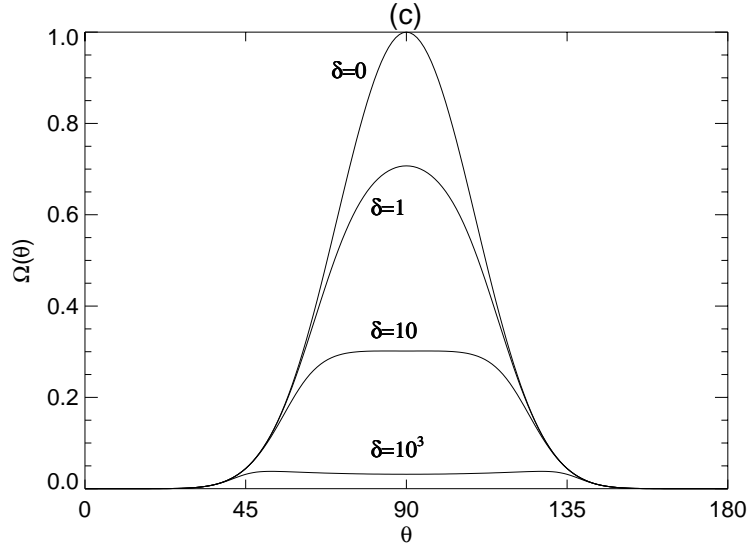

Fig. 2. Co-latitudinal dependence of the angular velocity of the roots of the field lines $\Omega(\theta)$ : in a) for $\delta=4$, in $\mathbf{b}$ ) for $\epsilon=2$, in c) for $\epsilon=10$

found if we write $M_{\mathrm{A}}=\left(\rho V_{\mathrm{r}}^{2} / 2\right) /\left(B_{\mathrm{r}}^{2} / 8 \pi\right)$, which represents the ratio of kinetic to magnetic energy density. If $M_{\mathrm{A}} \ll 1$ magnetic energy dominates, whereas if $M_{\mathrm{A}} \gg 1$ the dominant energy is kinetic. The radius $R_{*}$ represents the distance at which magnetic energy ceases to dominate over the kinetic energy. The regularity condition at $R_{*}$, $M_{\mathrm{A}}\left(R_{*}\right)=1$, constrains the two functions $L(\theta)$ and $\Omega(\theta)$ : $L(\theta)=\Omega(\theta) r_{*}^{2} \sin ^{2} \theta$.

If we now substitute Eqs. (20), (14) into Eqs. (8), (9), respectively, and compare the resulting expressions with 
(a)

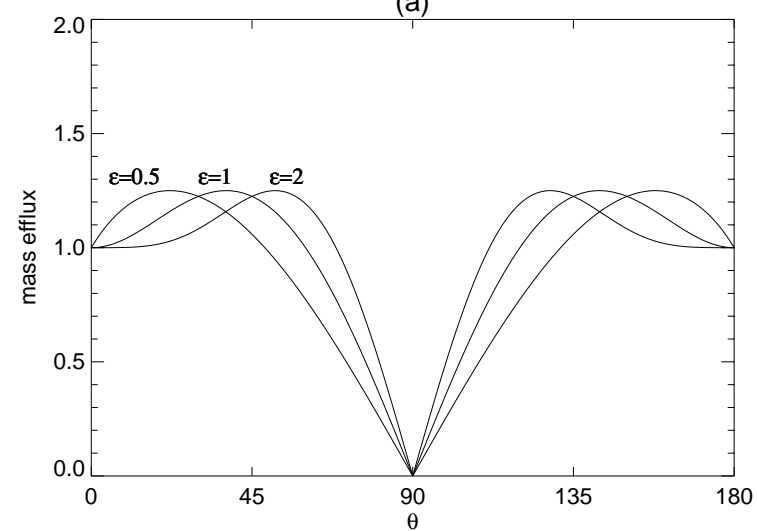

(b)

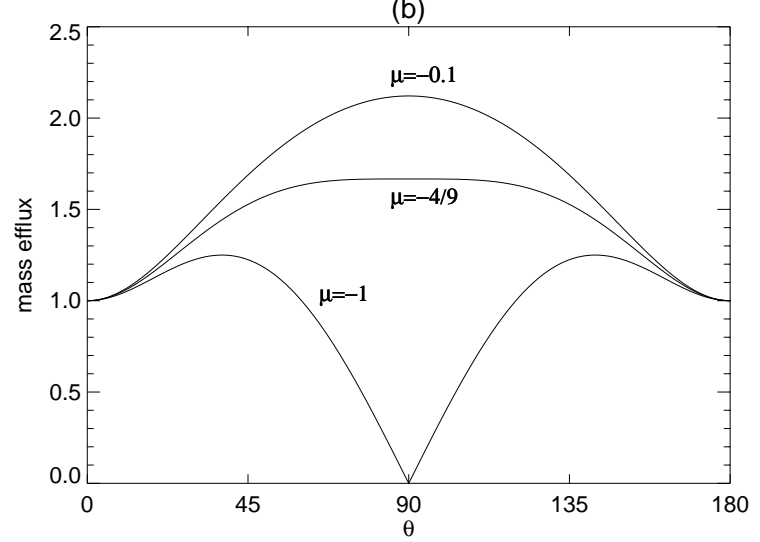

Fig. 3. Co-latitudinal dependence of the mass efflux $\dot{m}(\theta)$ for $\delta=4$ : in a) for $\mu=-1$, in $\mathbf{b}$ ) for $\epsilon=1$

Eqs. (26) and (27), we can deduce that $V_{0} B_{1}=V_{1} B_{0}$, together with the general form for $\Omega(\theta)$,

$\Omega(\theta)=\frac{\lambda V_{0} Y_{*}}{r_{0}} \frac{\sin ^{\epsilon-1} \theta}{\sqrt{1+\delta \sin ^{2 \epsilon} \theta}}$,

where $\lambda=V_{1} / V_{0}=B_{1} / B_{0}$ measures the ratio of azimuthal to radial velocities at the base of the wind, while

$L(\theta)=\frac{\lambda V_{0} r_{0}}{\left(M_{\mathrm{A}}^{0}\right)^{2}} \frac{\sin ^{\epsilon+1} \theta}{\sqrt{1+\delta \sin ^{2 \epsilon} \theta}}$.

The angular dependence of $\Omega(\theta)$ as the parameters $\epsilon$ and $\delta$ vary is worth some attention and is plotted in Fig. 2. This behaviour of $\Omega(\theta ; \epsilon, \delta)$ which emerges naturally from the separation of the variables in the governing equations can be compared, for example, with the rotation law of sunspots and solar photospheric magnetic fields. Except for the regions around the poles (since from Eq. (29), $\Omega(\theta)$ goes to zero for $\theta=0$ and $\left.180^{\circ}\right)$, the graphs of Figs. 2a-c for $\epsilon>1$ show similarities with the angular dependence of $\Omega(\theta)$ arising from the yearly averaged rotation profiles of photospheric magnetic fields (Snodgrass 1983).

\subsection{Mass and angular momentum efflux}

A quantity of interest is the mass efflux $\left(\rho V_{\mathrm{r}} r^{2}\right)$ or, equivalently, the mass loss rate per infinitesimal solid angle $\mathrm{d} \Sigma$

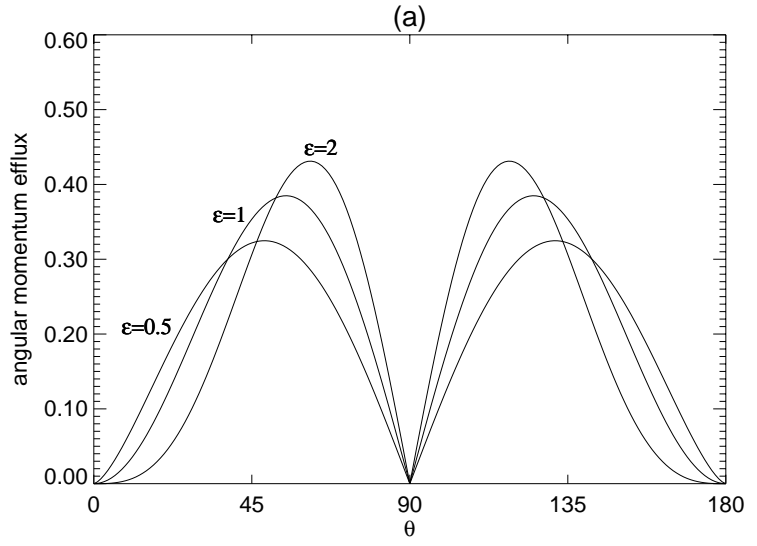

(b)

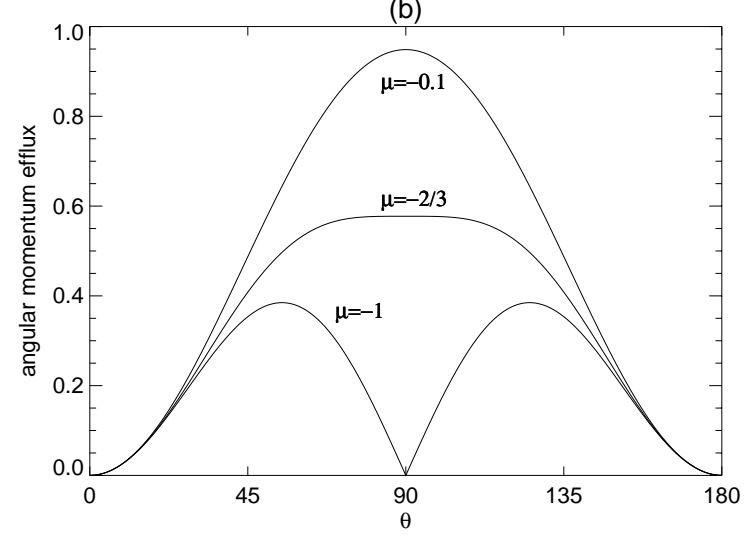

Fig. 4. Co-latitudinal dependence of the angular momentum efflux $i(\theta)$ : in a) for $\mu=-1$, in $\mathbf{b})$ for $\epsilon=1$

at the angle $\theta$ (see TT91). From the equation of conservation of mass Eq. (3) this has to be a function of $\theta$ alone, which we shall denote by $\dot{m}(\theta)$

$\dot{m}(\theta)=\rho_{0} V_{0} r_{0}^{2} \sqrt{\left(1+\mu \sin ^{2 \epsilon} \theta\right)\left(1+\delta \sin ^{2 \epsilon} \theta\right)}$.

Figure 3 shows the variation of the mass efflux with latitude. It vanishes at the equator only for $\mu=-1$. For a particular $\delta$, if $|\mu|<\delta /(2 \delta+1)$ the maximum of $\dot{m}$ occurs for $\theta=90^{\circ}$, while if $|\mu|>\delta /(2 \delta+1)$ it occurs for $0<\theta<90^{\circ}$. This is shown in Fig. $3 \mathrm{~b}$ for $\delta=4$. We simply note in passing that the first of these angular dependences of the mass loss is reminiscent of some observed intense mass loss rates that are thought to occur through equatorial stellar winds (e.g. from Be stars observed equator-on). Also, measurements of the Lyman alpha emission by the satellites Mariner 10, Prognoz, Ulysses and SoHO (Bertaux et al. 1997) have implied that there is higher mass efflux at the equator than at the pole.

We may next introduce the angular momentum efflux $\dot{l}(\theta) \equiv \rho V_{\mathrm{r}} r^{2} L(\theta)$ which is the angular momentum loss rate per infinitesimal solid angle $\mathrm{d} \Sigma$ at the angle $\theta$,

$\dot{i}(\theta)=\frac{\lambda \rho_{0} V_{0}^{2} r_{0}^{3}}{\left(M_{\mathrm{A}}^{0}\right)^{2}} \sin ^{\epsilon+1} \theta \sqrt{1+\mu \sin ^{2 \epsilon} \theta}$.

The variation of $i(\theta)$ with latitude is shown in Fig. 4. As with the mass efflux, the angular momentum efflux vanishes at the equator for $\mu=-1$. In TT91 the angular 
momentum efflux was assumed a priori, from which the simplest possible forms for the azimuthal hydromagnetic field were obtained.

\subsection{Hydromagnetic field and density}

The azimuthal components can now be obtained

$$
\begin{aligned}
& V_{\phi}(R, \theta)=\lambda V_{0} \frac{R \sin ^{\epsilon} \theta}{\sqrt{1+\delta \sin ^{2 \epsilon} \theta}}\left(\frac{Y_{*}-Y}{1-M_{\mathrm{A}}^{2}}\right), \\
& B_{\phi}(R, \theta)=\lambda B_{0} \frac{\sin ^{\epsilon} \theta}{R}\left(\frac{R^{2} / R_{*}^{2}-1}{1-M_{\mathrm{A}}^{2}}\right) .
\end{aligned}
$$

Note that $V_{\phi}$ is maximum at the equator. We recall that the radial hydromagnetic field and density are of the form

$$
\begin{aligned}
& V_{\mathrm{r}}(R, \theta)=V_{0} Y(R) \sqrt{\frac{1+\mu \sin ^{2 \epsilon} \theta}{1+\delta \sin ^{2 \epsilon} \theta}}, \\
& B_{\mathrm{r}}(R, \theta)=\frac{B_{0}}{R^{2}} \sqrt{1+\mu \sin ^{2 \epsilon} \theta} \\
& \rho(R, \theta)=\frac{\rho_{0}}{Y R^{2}}\left(1+\delta \sin ^{2 \epsilon} \theta\right) .
\end{aligned}
$$

\subsection{Balance of forces}

At this stage, we still have to deduce an equation for $Y(R)$, together with the form for the pressure $p(R, \theta)$ and hence the temperature $T(R, \theta)$. Going back to the $r$ - and $\theta$ components of the momentum equation - Eqs. (4) and (5) - and assuming that the variables $R$ and $\theta$ separate in these equations, we must have

$Q(R, \theta)=Q_{0}(R)+Q_{1}(R) \sin ^{2 \epsilon} \theta$,

where $Q(R, \theta)$ is the dimensionless pressure defined by

$p(R, \theta)=\frac{\rho_{0} V_{0}^{2}}{2} Q(R, \theta)$.

$Q_{0}$ represents the spherically symmetric part of the pressure, while $Q_{1}$ includes the effects of the anisotropy. Substitution of Eqs. (38), (39) into Eqs. (4) and (5) yields the following three equations for $Q_{0}(R), Q_{1}(R)$ and $Y(R)$

$$
\begin{aligned}
Q_{1}(R)= & -\frac{\mu}{\left(M_{\mathrm{A}}^{0}\right)^{2} R^{4}}+\frac{\lambda^{2}}{\epsilon Y}\left(\frac{Y-Y_{*}}{1-M_{\mathrm{A}}^{2}}\right)^{2} \\
& -\left(1+\frac{1}{\epsilon}\right) \frac{\lambda^{2}}{\left(M_{\mathrm{A}}^{0}\right)^{2} R^{2}}\left(\frac{1-R^{2} / R_{*}^{2}}{1-M_{\mathrm{A}}^{2}}\right)^{2}, \\
\frac{\mathrm{d} Q_{1}}{\mathrm{~d} R}= & -\frac{\delta \nu^{2}}{Y R^{4}}-\frac{2 \mu}{R^{2}} \frac{\mathrm{d} Y}{\mathrm{~d} R}+\frac{2 \lambda^{2}}{Y R}\left(\frac{Y-Y_{*}}{1-M_{\mathrm{A}}^{2}}\right)^{2} \\
& -\frac{\lambda^{2}}{\left(M_{\mathrm{A}}^{0}\right)^{2} R^{2}} \frac{\mathrm{d}}{\mathrm{d} R}\left(\frac{1-R^{2} / R_{*}^{2}}{1-M_{\mathrm{A}}^{2}}\right)^{2}, \\
\frac{\mathrm{d} Q_{0}}{\mathrm{~d} R}= & -\frac{\nu^{2}}{Y R^{4}}-\frac{2}{R^{2}} \frac{\mathrm{d} Y}{\mathrm{~d} R},
\end{aligned}
$$

in which $\nu$ is the ratio of the escape speed to the radial speed at the base of the outflow

$$
\nu=\frac{V_{\mathrm{esc}}}{V_{0}}=\frac{\sqrt{2 G M / r_{0}}}{V_{0}}
$$

To understand the interplay between different forces involved in the mechanism of this type of wind, let us describe one by one the various terms in the above equations. All of them are written so that the pressure gradient term is isolated on the left-hand side. Equation (40) represents the force equilibrium across the field lines. On the right-hand side of this, the various terms represent, respectively, the magnetic pressure, the centrifugal force and the magnetic tension. The last two equations express the equilibrium of forces along the radial direction. Equation (41) shows the anisotropic terms, namely, the anisotropic part of the gravitational and inertial forces, the centrifugal force and the magnetic tension term, respectively. The isotropic terms are shown in Eq. (42). These are related to the gravitational and inertial forces, respectively. Equations (40) and (41) can be combined to give a single expression for $Y(R)$

$\frac{\mathrm{d} Y}{\mathrm{~d} R}=\frac{F(R)}{G(R)}$

where

$$
\begin{aligned}
& F(R)=\frac{\delta \nu^{2}}{Y R^{4}}+\frac{4 \mu}{\left(M_{\mathrm{A}}^{0}\right)^{2} R^{5}}+\frac{2 \lambda^{2}}{\epsilon} \frac{Y}{R M_{\mathrm{A}}^{2}\left(1-M_{\mathrm{A}}^{2}\right)^{2}} \\
& \times\left[\frac{(1+\epsilon) M_{\mathrm{A}}^{2}-\epsilon}{M_{\mathrm{A}}^{2}} \frac{R^{4}}{R_{*}^{4}}-\left((2+\epsilon) M_{\mathrm{A}}^{2}-(1+\epsilon)\right)\right] \\
& G(R)=-\frac{2 \mu\left(M_{\mathrm{A}}^{0}\right)^{2} Y}{M_{\mathrm{A}}^{2}}-\frac{\lambda^{2}}{\epsilon\left(1-M_{\mathrm{A}}^{2}\right)^{2}} \\
& \times\left[\frac{2 M_{\mathrm{A}}^{2}-1}{M_{\mathrm{A}}^{4}} \frac{R^{4}}{R_{*}^{4}}-1\right] .
\end{aligned}
$$

The above differential equation for $Y(R)$ requires a boundary condition. For convenience, let us choose $Y(1)=1$, which defines $V_{0}=V_{\mathrm{r}}(R=1, \theta=0)$ from Eq. (35). From the classical ideal gas law we can now express temperature as

$$
T(R, \theta)=\frac{m V_{0}^{2} Y R^{2}}{2 k_{B}\left(1+\delta \sin ^{2 \epsilon} \theta\right)} Q(R, \theta) .
$$

\section{Parametric study of the solution}

\subsection{Critical points}

In order to determine the full solution we need to solve Eqs. (44), (45), (46) for $Y(R)$. This is a first-order nonlinear differential equation which can be integrated numerically, using a standard routine. A first inspection shows that this equation has a singular point at $R=R_{*}, Y=Y_{*}$, where $M_{\mathrm{A}}=1$, or in other words where the radial velocity equals the radial Alfvénic velocity $V_{\mathrm{A}}=B_{\mathrm{r}} / \sqrt{4 \pi \rho}$. The term singular point is used here in the sense that both the numerator and denominator must vanish there. 


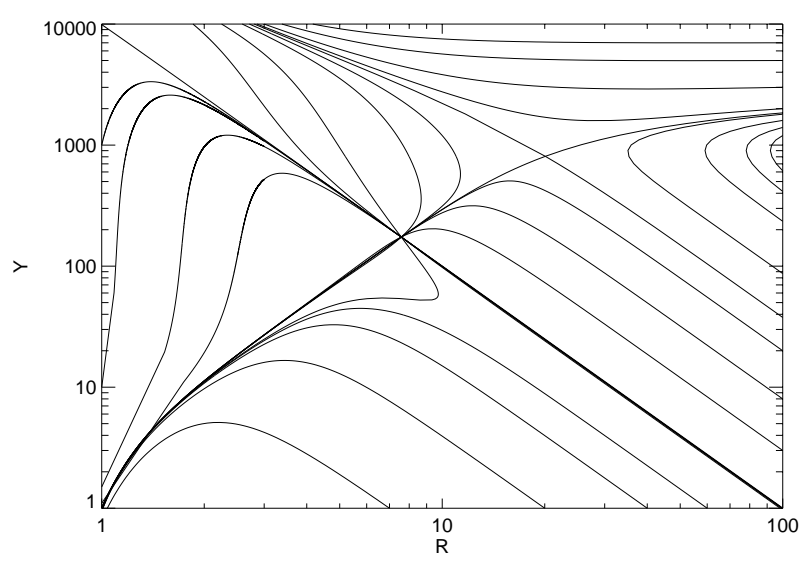

Fig. 5. Topology of the radial dependence of the radial velocity $Y(R)$ for a solar-type highly magnetized star with $\lambda=0.5$, $\nu=120, M_{\mathrm{A}}^{0}=0.01, \delta=4, \epsilon=1$ and $\mu=-0.1$. Note the presence of both critical points

In a more general geometry with meridional components, this point, known as the Alfvénic point, corresponds to the location where the poloidal speed equals the poloidal Alfvén speed. It is present in magnetic wind models (cf. Weber \& Davis 1967; Mestel 1968) and is a consequence of the steady-state assumption. It delimits the magnetically dominated region beyond which the torque exerted by the magnetic field ceases to dominate over the angular momentum carried by the fluid. The exact position of this point cannot be found analytically in this case, which complicates any type of numerical treatment as we shall see later. In a similar problem, TT91 find that expanding up to fourth order around $\left(R_{*}, Y_{*}\right)$, all slopes are allowed. We are thus in the presence of an improper node or star-type point. This means that no particular solution is filtered out by the presence of this singular point.

There is a second singular point, say at $\left(R_{\mathrm{X}}, Y_{\mathrm{X}}\right)$, found by satisfying simultaneously $F=G=0$ in Eq. (44). A first-order analysis around this point shows that only two slopes are allowed, one positive and one negative, giving rise to a saddle or $\mathrm{X}$-type point. Thus, this point is also a critical point in the sense that it chooses a critical solution. In a recent work, Tsinganos et al. (1996) have shown that, in general, at the critical points of axisymmetric and self-similar solutions, the component of the flow velocity perpendicular to the directions of axisymmetry (the $\phi$ direction in this model) and self-similarity (the $\theta$-direction in this model), equals the characteristic slow/fast MHD wave speed in that direction,

$V_{\mathrm{r}}^{4}-V_{\mathrm{r}}^{2}\left(V_{\mathrm{a}}^{2}+C_{\mathrm{s}}^{2}\right)+C_{\mathrm{s}}^{2} V_{\mathrm{a}, \mathrm{r}}^{2}=0$

with $C_{\mathrm{s}}$ the sound speed and $V_{\mathrm{a}}, V_{\mathrm{a}, \mathrm{r}}$ the total and radial components of the Alfvén speeds, respectively. The above equation is the equation for the speed of an MHD wave propagating in the $r$-direction.

Thus, at the critical point where Eq. (48) is satisfied, the $r$-component of the flow speed equals to the slow or the fast MHD mode wave speed in that direction. Now, in low magnetisation plasmas and in the case of parallel wave propagation, the slow mode wave speed coincides
Table 1. Location of both singular points for $\lambda=0.5, \nu=120$, $\delta=4:$ in a) for $M_{\mathrm{A}}^{0}=0.1$ and in $\mathbf{b}$ ) for $M_{\mathrm{A}}^{0}=0.01$

\begin{tabular}{llcc}
\hline$\epsilon$ & \multicolumn{1}{c}{$\mu$} & $R_{*}$ & $R_{X}$ \\
\hline 0.5 & -1 & 1.312 & 1.385 \\
1 & -1 & 1.248 & 1.285 \\
2 & -1 & 1.210 & 1.229 \\
1 & -0.1 & 1.157 & 1.168 \\
1 & -0.01 & 1.148 & 1.155 \\
1 & -0.001 & 1.147 & 1.153 \\
\hline \multicolumn{4}{c}{ (a) }
\end{tabular}

\begin{tabular}{llrl}
\hline$\epsilon$ & \multicolumn{1}{c}{$\mu$} & \multicolumn{1}{c}{$R_{*}$} & \multicolumn{1}{c}{$R_{X}$} \\
\hline 0.5 & -1 & 10.064 & 24.220 \\
1 & -1 & 10.550 & 21.862 \\
2 & -1 & 11.038 & 19.577 \\
1 & -0.1 & 7.581 & 20.084 \\
1 & -0.01 & 7.429 & 28.297 \\
1 & -0.001 & 7.413 & 41.173 \\
\hline \multicolumn{4}{c}{ (b) }
\end{tabular}

with the Alfven speed. On the other hand, in high magnetization plasmas and in the case of parallel wave propagation, it is the fast mode wave speed that coincides with the Alfven speed. In the present case we have a degeneracy in the sense that there are only two critical surfaces in view of the assumption that the critical surfaces are spherical (which seems to be a good assumption, at least in the case of the solar wind - Exarhos \& Moussas 2000). Note that due to the non-polytropic assumption, the sound speed is ill-defined and we cannot easily determine whether we are in a low- or high-magnetisation regime. We conjecture that here we have a situation analoguous to low magnetisation plasmas and at the X-type critical point the superAlfvenic radial flow speed equals to the radial component of the fast MHD mode wave speed, while the slow critical transition coincides everywhere with the Alfvénic transition because of self-similarity.

We should stress at this stage that both the Alfvénic point $\left(R_{*}, Y_{*}\right)$ and this $\mathrm{X}$-type point are (loosely) called equilibrium or critical points since they satisfy the regularity condition $F(R)=G(R)=0$. However, since the first one does not filter any solution (i.e. all slopes are allowed through it), while the second one (as for any saddle point) selects a particular solution sometimes referred to as the critical solution, only the X-type singularity corresponds to a true critical point.

We performed a numerical integration of Eqs. (44), (45), (46). The general topology is shown in Fig. 5 for a highly magnetized medium and for representative values of $\mu$ and $\epsilon$. The position of the critical points was found by an iterative procedure. The integration yields a single solution crossing the $\mathrm{X}$-type point with positive slope and satisfying the boundary condition $Y(1)=1$. For this critical solution, the flow starts near the star with low speeds and connects to large distances where it attains large super-Alfvénic velocities. There is another critical solution, which crosses the X-type point with negative slope and is always decelerating. There is a limiting value of $\epsilon$ above which we couldn't find a wind solution satisfying the boundary condition $Y(1)=1$. For example, if $\lambda=0.5, \nu=120$ and $\delta=4$, there is no solution for $\epsilon \gtrsim 5$, if $M_{\mathrm{A}}^{0}=0.1$ and for $\epsilon \gtrsim 2$, if $M_{\mathrm{A}}^{0}=0.01$.

Once the positions of both $R_{*}$ and $R_{\mathrm{X}}$ are known, all other types of solutions can be easily found. They include breeze-type solutions that cross the Alfvénic point but not the X-type point and reach sub-Alfvénic asymptotic speeds for large distances. 
(a)

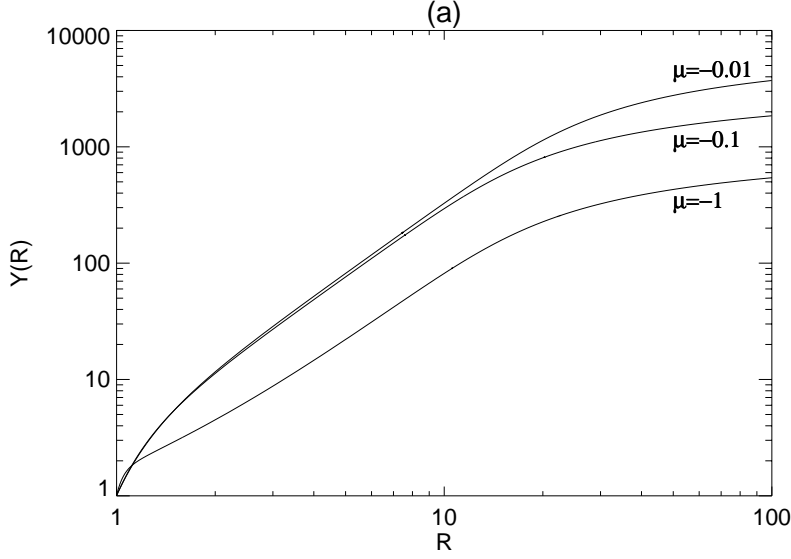

(b)

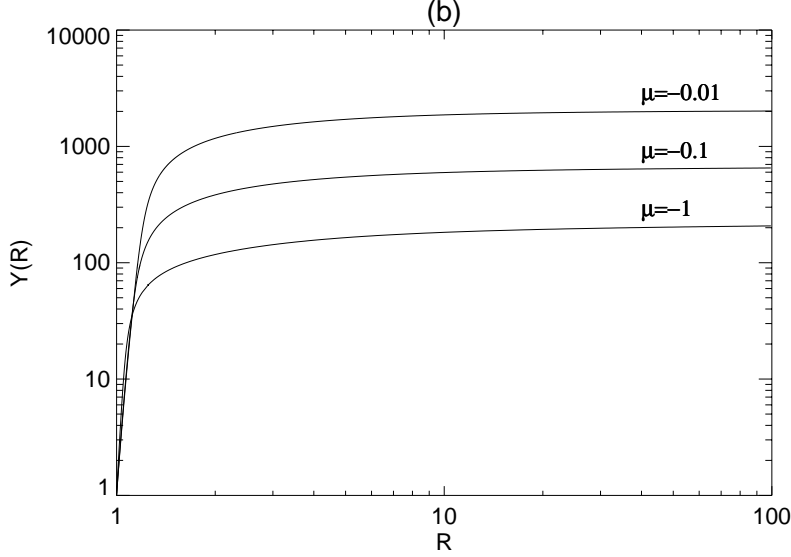

Fig. 6. Radial dependence of the dimensionless radial velocity $Y(R)$ for a solar-type star with $\lambda=0.5, \nu=120, \delta=4, \epsilon=1$ : in a) for $M_{\mathrm{A}}^{0}=0.01$, in b) for, $M_{\mathrm{A}}^{0}=0.1$

Table 1 shows the positions of both singular points for $M_{\mathrm{A}}^{0}=0.1$ and $M_{\mathrm{A}}^{0}=0.01$ and different values of $\epsilon$ and $\mu$. We have taken $\lambda=0.5, \nu=120$ and $\delta=4$.

\subsection{Radial velocity}

The initial acceleration decreases as $M_{\mathrm{A}}^{0}$ decreases, Figs. 6a,b, and also as $\lambda$ increases. In other words, the more the outflow is magnetised and rotating, the smaller is the initial acceleration and both the magnetic field and rotation are inhibiters of the initial acceleration of the outflow. This may be understood using a simple argument, as follows. Assume without loss of generality that $\delta=0$, such that the weight of a parcel of plasma is the same at any angle $\theta$, at some fixed radial distance $R$. Also for simplicity assume that $\mu=-1$ and $\epsilon=1$. Then, because of the sinusoidal dependence of the radial flow speed with the latitude, at the equator we have static conditions such that the weight of the plasma is balanced there mainly by the centrifugal force and the radial component of the Lorenz force (the centrifugal force is always positive, i.e., outwards, while in most of the cases examined the radial component of the magnetic force is also positive). Then, moving at the pole and at the same radial distance $R$, we find the same inwards gravitational plasma weight (since (a)

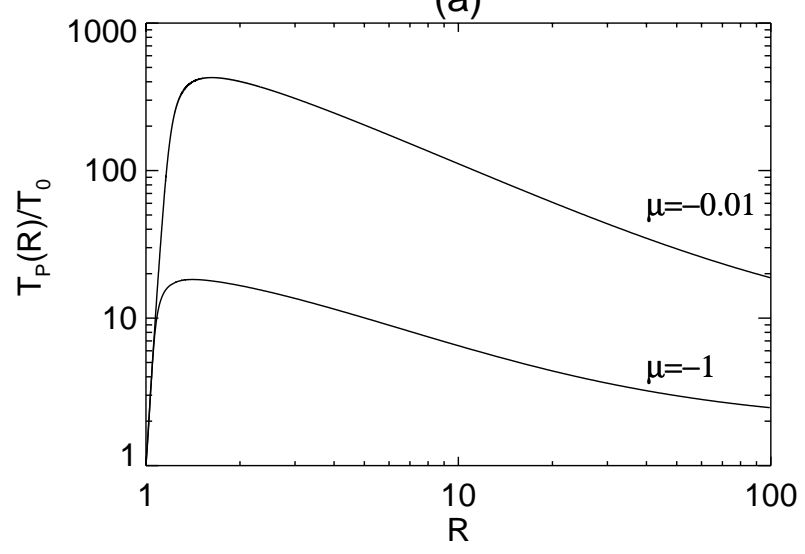

(b)

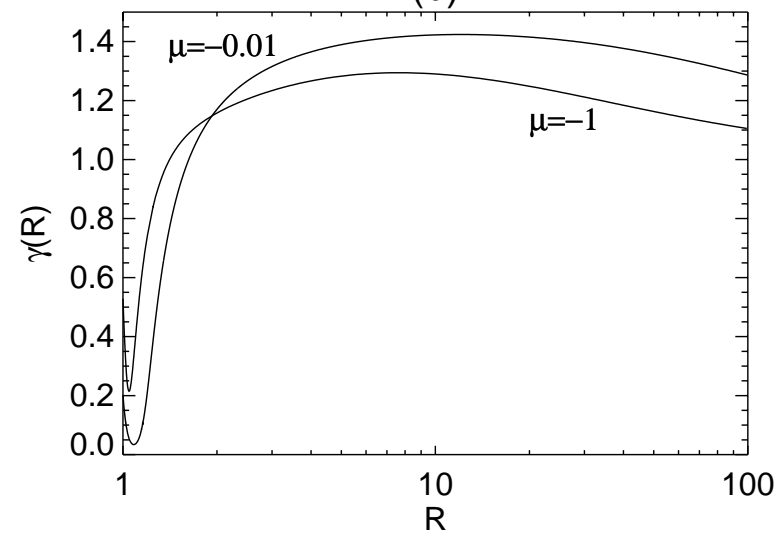

Fig. 7. Radial dependence of: a) the dimensionless temperature at the pole $\left.T_{\mathrm{P}}(R) / T_{0}, \mathbf{b}\right)$ the polytropic index $\gamma(R)$, both for $\lambda=0.5, \nu=120, M_{\mathrm{A}}^{0}=0.1, \delta=4, \epsilon=1$ and two values of $\mu$

$\delta=0$ ), but no centrifugal and radial magnetic forces acting there due to their $\sin ^{2} \theta$ dependence. The net result is a decelerating force, relatively to the cases $M_{\mathrm{A}}^{0}=\lambda=0$. For a mildly magnetized case $\left(M_{\mathrm{A}}^{0}=0.1\right)$, Fig. $6 \mathrm{~b}$ shows that the initial acceleration is very high and the radial velocity rapidly attains its asymptotic value.

The asymptotic form of $Y(R)$ for large $R$ can be obtained after integrating Eq. (44)

$Y^{3} \simeq 3\left(1+\frac{1}{\epsilon}\right) \frac{\lambda^{2}}{\left(M_{\mathrm{A}}^{0}\right)^{6}|\mu| R_{*}^{4}} \ln R$

For $M_{\mathrm{A}}^{0} \ll 1$, the asymptotic speed increases logarithmically with $R$, and $Y$ increases with a decrease of $M_{\mathrm{A}}^{0}$ or a decrease of $\epsilon$ and $|\mu|$. When $M_{\mathrm{A}}^{0}>1$, the logarithmic derivative $\mathrm{d} Y^{3} / \mathrm{d} \ln R$, for large $R$, is very small (behaving like $\left(M_{\mathrm{A}}^{0}\right)^{-6}$ ), and $Y$ can be taken as constant. The flow is hydrodynamically dominated from $R=1$, while both singular points, $R_{*}$ and $R_{\mathrm{X}}$, almost coincide in the region $R<1$ (i.e., below the base of the wind). We recall that in the hydrodynamic case (Paper I), $Y$ approaches rapidly a constant value, independent of $\epsilon$.

The cautious reader may have already noted that in Eq. (49) the asymptotic radial speed is the product of a logarithmically diverging isothermal wind speed which 
(a)

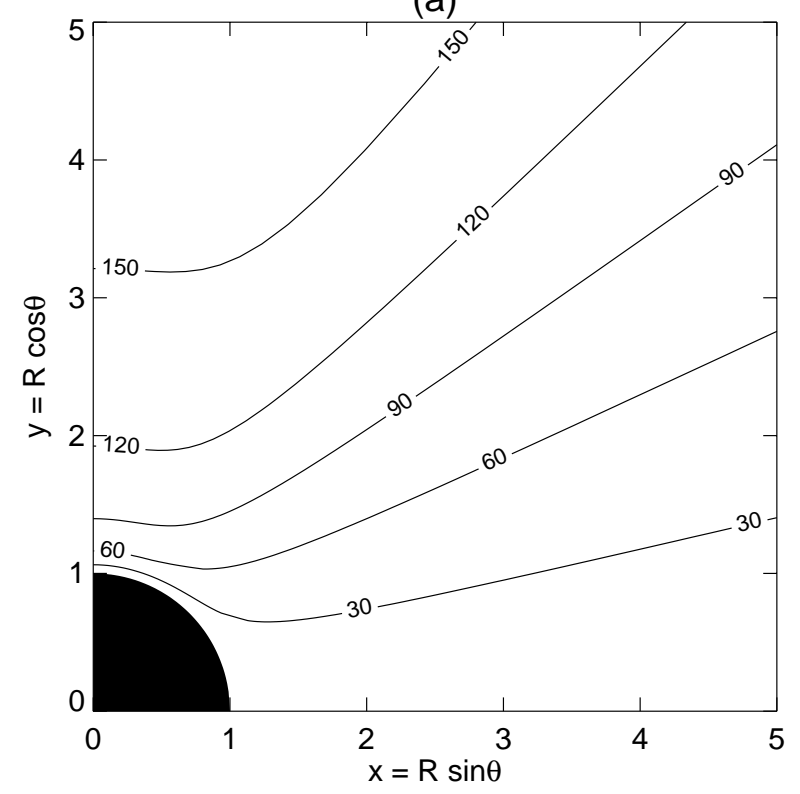

(b)

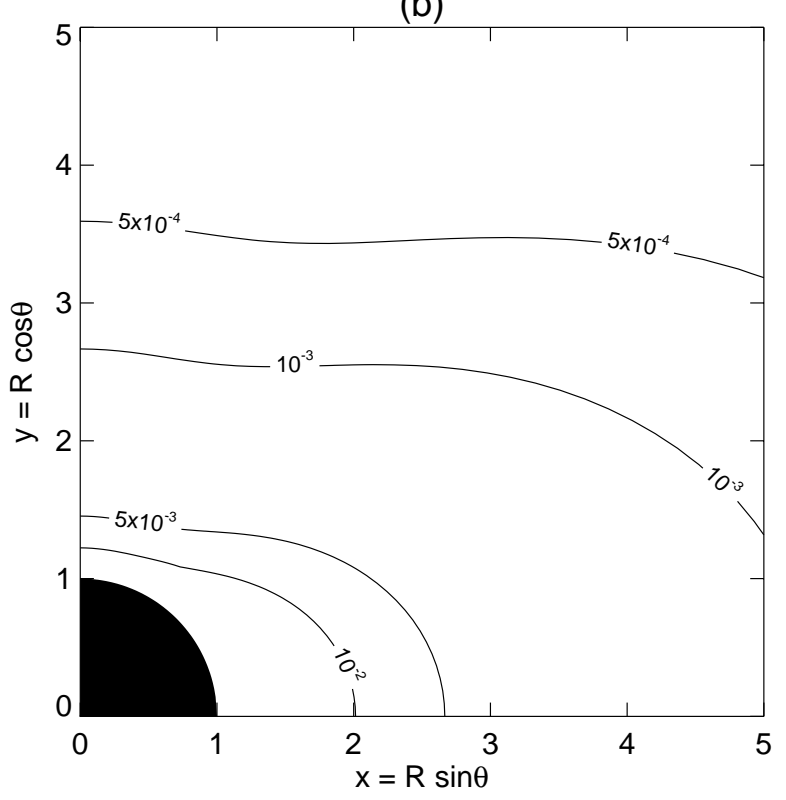

Fig. 8. Contour plots of: a) the radial velocity $V_{\mathrm{r}}(R, \theta) / V_{0}$ and $\mathbf{b}$ ) the density $\rho(R, \theta) / \rho_{0}$, for $\lambda=0.5, \nu=120, M_{\mathrm{A}}^{0}=0.1$, $\delta=4, \epsilon=2, \mu=-1$

increases like $(\ln R)^{1 / 3}$ (see TT91) and Michel's characteristic speed $\left(\Omega^{2} F_{\mathrm{B}}^{2} / \dot{M}\right)^{1 / 3}$, where $F_{\mathrm{B}}$ and $\dot{M}$ are the magnetic and mass fluxes (MacGregor 1996). This is also checked by the fact that the effective polytropic index $\gamma(R \longrightarrow \infty) \longrightarrow 1$, as seen in Fig. 7 .

\subsection{Temperature and effective polytropic index}

In this study by not using a polytropic relationship between pressure and density $\left(p \propto \rho^{\gamma}\right.$, with $\gamma$ the constant polytropic index) we avoided constraining the exchange of energy so as to keep $\gamma$ constant. In our approach, we can define an effective polytropic index given by

$\gamma \equiv\left[\frac{\partial \ln p}{\partial \ln \rho}\right]_{A=\text { const. }}$

for each field line $A(\theta)=$ const. (Weber 1970, TT91). This effective polytropic index is no longer a constant, but instead a function of $R$. The form of $\gamma(R)$ is closely associated with the variation of temperature with distance from the central object. In particular, if $\gamma<1$ there is intense heating and the temperature increases, whereas if $\gamma>1$ there is a depletion of heating and the temperature decreases (see Fig. 7).

In Fig. 8 we have chosen to illustrate the twodimensional character of the solution deduced in this paper, with parameters corresponding to a mildly magnetized object $\left(M_{\mathrm{A}}^{0}=0.1\right)$, by plotting the latitudinal dependence of the radial velocity and density. The velocity, is higher at the polar axis while the density is maximum at the equator. The angular dependence of these quantities is similar to the latitudinal distribution of the density and velocity in coronal holes.

\section{Discussion}

We have explored in this paper a new set of exact solutions of the steady axisymmetric MHD equations relevant to stellar wind problems.

Analogously to the case with no magnetic field (Paper I), we were able to obtain the latitudinal dependences of the different variables and these involve only the anisotropy parameters, $\delta, \epsilon$ and $\mu$. The parameter $\delta$ is associated with the ratio of equatorial to polar density, $\epsilon$ controls the width of the density and velocity profiles for a fixed variation between pole and equator, while $\mu$ is connected to the latitudinal anisotropy of the distribution of radial kinetic energy density. The other three parameters of the problem, which come about in the solution for the radial functions, are $\lambda$, which controls the strength of rotation, $\nu$, a measure of the escape speed from the central object and $M_{\mathrm{A}}^{0}$ which is related the strength of the magnetic pressure.

The degree of collimation, together with the anisotropy in the density distribution, is effectively controlled by $\delta, \epsilon$ and $\mu$. These solutions could be pertinent to the plasma dynamics in polar coronal holes, stellar and extragalactic jets and even star-forming regions. The solution with $\mu=-1$ has zero radial velocity at the equator and a continuous radial magnetic field across the equator. A discontinuity and associated current sheet arises for $\mu \neq-1$. Also, the angular velocity of the roots of the field lines at the surface of the central object is such that it resembles qualitatively solar observations only for $\epsilon>1$.

The topology of the solutions is controlled by two distinct singular points. The first one is the usual Alfvénic point and corresponds to the location where the radial velocity of the outflow equals the radial Alfvénic velocity. All solutions pass through this high-order singularity and it corresponds to an improper node or star-type point. 
There is a critical point present downstream from this Alfvén transition. It is an X-type critical (or saddle) point which is responsible for filtering a single solution corresponding to a vanishing pressure at infinity the wind-type solution. In a similar problem, Tsinganos et al. (1996) have shown that at this new critical point, the $r$-component of the flow speed equals the slow/fast MHD mode wave speeds in that direction.

Because the field/streamlines are constrained to keep a helicoidal geometry, the pressure gradient for large distances has to be sufficiently large to balance the dominant tension force. Thus, in some cases the solution is not valid outside a cone around the polar axis. This limitation disappears for $M_{\mathrm{A}}^{0} \geq 0.05$.

There is a drastic change in the nature of the solution from high to lower values of $M_{\mathrm{A}}^{0}$. The solutions for high $M_{\mathrm{A}}^{0}$ manifest all the characteristics of a typical hydrodynamic wind, namely very large acceleration at the base of the atmosphere and temperature decaying very rapidly with distance. The magnetically dominated cases $\left(M_{\mathrm{A}}^{0} \ll 1\right)$ show lower acceleration at the base of the atmosphere and an almost isothermal atmosphere at larger distances.

The density anisotropy $(\delta)$ greatly favours the acceleration of the wind close to the base, while the strength of rotation $(\lambda)$ and the gravitational field $(\nu)$ slows down the initial speed. The solution far away from the source seems to be quite insensitive to $\epsilon$. The influence of $\mu$, on the other hand, is such that a decrease of $|\mu|$ not only diminishes the relative importance of the magnetic effects by decreasing the size of the magnetic lever arm, $R_{*}$, but also increases the value of the initial acceleration as well as the asymptotic radial velocity at large distances. The asymptotic plasma temperature increases for lower values of $|\mu|$.

This simple but self-consistent two-dimensional solution of the MHD equations has nevertheless some limitations. The assumption of separation of variables could be dropped, although we should not forget the nonlinear character of the governing coupled partial differential equations. The assumption of the neglect of the meridional components of the magnetic field and flow $\left(V_{\theta}, B_{\theta}\right)$ which limits their possible applications, could also be eliminated within the framework of the method of separation of the variables. Finally, as we've already discussed, the present investigation does not take into account the detailed energetics in the outflow. Nevertheless, the present study indicates that inherently non-spherically symmetric solutions of the MHD equations which do not have the previous limitations may exist. This ambitious undertaking to find them remains a challenge for the future.

Acknowledgements. J. Lima wishes to thank C. Sauty for his help and support and also the staff at FORTH in Crete for their kind hospitality. This work was supported by Programa Praxis XXI of the Fundação para a Ciência e a Tecnologia, by funds from the UK Science and Engineering Research Council, the Erasmus Programme and the EEC RT Network HPRNCT-2000-00153.

\section{References}

Bertaux, J. L., Quemerais, E., Lallement, T., et al. 1997, in The Corona and Solar Wind near Minimum Activity (ESA SP-404), 29

Biretta, J. A. 1996, in Solar and Astrophysical MHD Flows, ed. K. Tsinganos (Kluwer Academic Publishers), 357

Exarhos, G., \& Moussas, X. 2000, A\&A, 356, 315

Feldman, W. C., Phillips, J. L., Barraclough, B. L., \& Hammond, C. M. 1996, in Solar and Astrophysical MHD Flows, ed. K. Tsinganos (Kluwer Academic Publishers), 265

Giordano, S., Antonucci, E., Noci, G., Romoli, M., \& Kohl, J. L. 2000, ApJL., preprint [astro-ph/0001257]

Habbal, S. R., \& Tsinganos, K. 1983, J. Geophys. Res., 88, 1965

Hansteen, V. H., Leer, E., \& Holzer, T. E. 1997, ApJ, 482, 498

Hu, Y. Q., \& Low, B. C. 1989, ApJ, 342, 1049

Keppens, R., \& Goedbloed J. P. 1999, A\&A, 343, 251

Krasnopolsky, R., Li, Z-Y., \& Blandford, R. 1999, ApJ, 526, 631

Kopp, R. A., \& Holzer, T. E. 1976, Solar Phys., 49, 43

Leer, E., \& Holzer, T. E. 1980, J. Geophys. Res., 85, 4681

Lima, J. J. G., \& Priest, E. R. 1993, A\&A, 268, 641

Low, B. C., \& Tsinganos, K. 1986, ApJ, 302, 163

MacGregor, K. B. 1996, in Solar and Astrophysical MHD Flows, ed. K. Tsinganos (Kluwer Academic Publishers), 301

Mestel, L. 1968, MNRAS, 138, 359

Nerney, S. F., \& Suess, S. T. 1975, ApJ, 196, 837

Ouyed, R., \& Pudritz, R. E. 1997, ApJ, 482, 712

Parker, E. N. 1958, ApJ, 128, 664

Parker, E. N. 1963, in Interplanetary Dynamical Processes (Interscience Publishers, New York)

Pneumann, G. W., \& Kopp, R. A. 1971, Solar Phys., 18, 258

Priest, E. R. 1982, Solar Magnetohydrodynamics (D. Reidel, Holland)

Sakurai, T. 1985, A\&A, 152, 121

Sauty, C., \& Tsinganos, K. 1994, A\&A, 287, 893

Sauty, C., Tsinganos, K., \& Trussoni, E. 1999, A\&A, 348, 327

Snodgrass, H. B. 1983, ApJ, 270, 288

Steinolfson, R. S. 1988, J. Geophys. Res., 93, 14261

Steinolfson, R. S., Suess, S. T., \& Wu, S. T. 1982, ApJ, 255, 730

Suess, J., Wang, A. H., \& Wu, S. T. 1996, J. Geophys. Res., 101,19957

Trussoni, E., \& Tsinganos, K. 1993, A\&A, 269, 589

Trussoni, E., Tsinganos, K., \& Sauty, C. 1997, A\&A, 325, 1099

Tsinganos, K., \& Bogovalov, S. 2000, A\&A, 356, 989

Tsinganos, K., \& Low, B. C. 1989, ApJ, 342, 1028

Tsinganos, K., \& Trussoni, E. 1991, A\&A, 249, 156 (TT91)

Tsinganos, K., Sauty, C., Surlantzis, G., Trussoni, E., \& Contopoulos, J. 1996, in Solar and Astrophysical MHD Flows, ed. K. Tsinganos (Kluwer Academic Publishers), 427

Ustyugova, G. V., Koldoba, A. V., Romanova, M. M., Chechetkin, V. M., \& Lovelace, R. V. E. 1999, ApJ, 516, 221

Wang, A. H., Wu, S. T., Suess, S. T., \& Poletto, G. 1998, J. Geophys. Res., 103(A2), 1913

Weber, E. J. 1970, Solar Phys., 14, 480

Weber, E. J., \& Davis, L. J. 1967, ApJ, 148, 217 